\section{Pressure reconstruction by heterogeneous compression textiles}

\author{
Rong Liu \\ Institute of Textiles and Clothing, \\ The Hong Kong Polytechnic University, \\ Hung Hom, Kowloon, Hong Kong
}

\begin{abstract}
The homogeneous elastic structures of traditional compression modalities (e.g. compression stockings) generate uncontrolled cross-sectional pressures around leg geometrics, resulting in highly uneven pressure distributions, which could cause side effects and lowered user's compliance in compression therapy. A new type of heterogeneous compression sleeves (HCSs) has been developed using advanced 3D seamless knitting techniques to proactively reconstruct leg cross-sectional pressures. Pressure assessment was conducted in vivo on 20 healthy subjects' lower limbs applying the designed HCSs with hybrid elastic moduli when they were being mounted to the lower limbs under the two testing conditions (I\&II). The results demonstrated that the HCSs were capable to reshape compression around lower limbs with reduction of peak pressures at anterior tibia crest and increased pressures at posterior gastrocnemius regions. More even cross-sectional pressure profiles were achieved through reducing the anterior-posterior pressure differences at calf by $41.7-57.1 \%$ in condition II. This study attempts to reform source of pressure in mono-layered compression shell by the design of fabric structure itself rather than additional padding or foam insertions, to promote pressure function and user compliance in practice.
\end{abstract}

\section{Introduction}

Chronic venous insufficiency (CVI) is a long-term progressive condition, in which venous pooling reduces venous return, resulting in pain, itching, tiredness, varicose veins and even ulceration in lower limb, which affects approximate $25-40 \%$ of women and $10-20 \%$ of men globally ${ }^{1}$. Compression therapy has been used in CVI treatment for centuries. ${ }^{2}$ Textile-based compression interventions, as the essential source of pressure, have affected effectiveness of pressure dosage delivery, ${ }^{3,4}$ in which uniaxial gradient pressure is a core princi- ple of compression textiles used in CVI treatment. Through controlling dimensions and densities in continuous fabric segments along the compression shells, a degressive or progressive gradient fashion following certain residual pressure ratios have demonstrated to reduce venous hypertension or increase venous ejection. ${ }^{5-8}$ However, in clinical practice, high noncompliance resulting from discomfort affected their effectiveness, which was considered much underestimated by previous studies. ${ }^{9-11}$

Homogenous fabric structures were employed in traditional compression modalities (e.g. elastic compression stockings (ECSs), which passively generated skin pressures by stretching compression shells onto irregular limbs with larger sizes, as a result, the magnitudes of skin pressure at any angular sites around the limb largely depends upon the geometric structures of the anatomic sites located, causing highly uneven and uncontrollable pressure distributions ${ }^{12}$ and side effects, ${ }^{13}$ e.g. ischemia, necrosis and even ulcerations at bony prominence, especially for the elderly with thin and fragile skin. ${ }^{14}$ To date, few studies have looked at solutions aimed at reforming compression shell itself. Aim of this study is to explore a new approach to proactively reshape cross-sectional pressures using hybrid elastic knitting segments in a monolayered compression shell for achieving more even cross-sectional pressure distributions, which integrated with longitudinal gradient pressure as to achieve a new biaxial pressure control for improvement of CVI treatment.

\section{Materials and Methods}

The HCSs specimens were fabricated by using two types of interlaced Lycra covered polyamide elastomers, including inlay threads with linear densities of 260 deniers and ground knitting threads with linear densities of 40 deniers. Through applying advanced 3D seamless knitting techniques, hybrid elastic segments were formed using specially designed full-knit and laid-in knitting structures. Each HCS shell included 8 seamlessly knitted segments. A total of nine types of segments with hybrid ratios of high-low elastic moduli (i.e. shorter or longer stretch) were designed, including: i) HCS-A: 8:0, i.e. all segments were set with low elastic moduli presenting longer stretch property, which was similar with traditional elastic compression stocking); ii) HCSs-B, C, D, E, F, G and H: specimens with hybrid elastic moduli. The proportions of segments
Correspondence: Rong Liu, Institute of Textiles and Clothing, The Hong Kong Polytechnic University, Hung Hom, Kowloon, Hong Kong.

E-mail: rong.liu@polyu.edu.hk

Acknowledgements: we would like to thank the Hong Kong Polytechnic University to support this study through research projects 1ZE7K, G-YBUY and ITF project ITS/031/17.

Conference presentation: International Compression Club (ICC) Meeting, Paris, 2017.

This work is licensed under a Creative Commons Attribution 4.0 License (by-nc 4.0).

(C) Copyright R. Liu 2018

Licensee PAGEPress, Italy

Veins and Lymphatics 2018; 7:7621

doi:10.4081/vl.2018.7621

with low-high elastic moduli were set at $7: 1$ (B), 6:2(C), 5:3(D), 4:4(E), 3:5 (F), 2:6(G) and $1: 7(\mathrm{H})$, respectively; and iii) HCS-I: $0: 8$, i.e. all segments were set with high elastic moduli, presenting shorter stretch property, which was similar with rigid or semi-rigid bandage. Skin pressures exerted by the developed HCSs were tested under the two conditions, including: i) condition I: mounting the short-stretch segments (with higher elastic moduli) at anterior tibia crest, and the long-stretch segments (with lower elastic moduli) at posterior elastic muscular region; and ii) condition II: mounting the long-stretch segments at the anterior tibia and short-stretch segments (with higher elastic moduli) at posterior elastic muscular calf. A total of 20 healthy subjects aged $24.0 \pm 1.6$ years old (weight: $59.2 \pm 10.4 \mathrm{~kg}$, height: $165.5 \pm 10.5 \mathrm{~cm}$ and body mass index $21.6 \mathrm{~kg} / \mathrm{m}^{2}$ ) participated in the pressure assessment in vivo. Their calves with maximum circumferences were $34.3 \pm 2.7 \mathrm{~cm}$. The stretch ratio of HCS fabrics was up to approximate. The skin pressures around the calf cross-sections (anterior P1, medial P2, posterior P3 and lateral $\mathrm{P} 4$ ) were assessed using Picopress ${ }^{\circledR}$ testing system (Microlab Italia, Padua, Italy) when subjects being worn with HCSs. Each compression shell was measured by three times and pressure values on average were recorded.

\section{Results}

Figure 1A presents the skin pressure variations around the calf exerted by the nine designed HCSs under the two testing conditions. It can be seen that the increase of 
shorter stretch segments increased the overall skin pressures for both conditions. In general, the pressure differences around the calf were increased when the shorter stretch segments were placed at anterior rigid tibia crest in condition I. On the contrary, the anterior-posterior pressure differences were obviously reduced by $41.7-57.1 \%$ in condition II, especially for the specimens F (3:5) and $\mathrm{G}(2: 6)$. Figure $1 \mathrm{~B}$ illustrates the reshaped pressures around the four directions of the calves under both conditions. The hybrid elastic design did not significantly influence skin pressures at medial and lateral lower limb, but did reduce the anterior peak focal pressures and increased posterior muscular pressures at calf. This newly reconstructed pressure profile is promising to avoid side effects caused by excessive pressure and to augment muscular pumping action in dynamic wear. Based on this testing results, a new generation of compression stocking with bi-axial pressure profile has been developed (Figure 2), which produced degressive gradient pressure from ankle to knee in a sagittal plane and more even crosssectional pressures around lower limbs in a horizontal plane.

\section{Discussion}

Shorter stretch materials (e.g. static stiffness index SSI $>10 \mathrm{mmHg} / \mathrm{cm}$ ) deliver intermittent high-working but low-resting pressures, which has demonstrated a significant muscular pumping action for promoting venous return in previous clinical studies. However, the sustained uneven pressures by compression shells, and the short functional term (e.g. one-week) by short-stretch fabrics reduced their user compliance and usability in practice. The available studies on heterogeneous properties of compression textiles mainly focused on the four aspects, i) yarn multi-components; ii) anisotropic and nonlinear behaviors of elastic fabrics; iii) integrative elastic or inelastic layers in sub-bandage; and iv) tension variations by inserting or sewing rigid or supportive elements to elastic fabrics. In fact, combining hybrid elastic moduli materials into a continuous compression shell is challenging in fabrication since any protruding seams or overlaps may potentially alter local pressure and chafe the skin in long-term wear. This study integrated high and low elastic moduli segments into mono-layered compression shell by applying seamless laid-in structures and adjusting yarn tension and knitting densities to produce a bi-axial pressure profile along the lower limb. According to Laplace's Law, ${ }^{15}$ the reduced fabric tension may lower
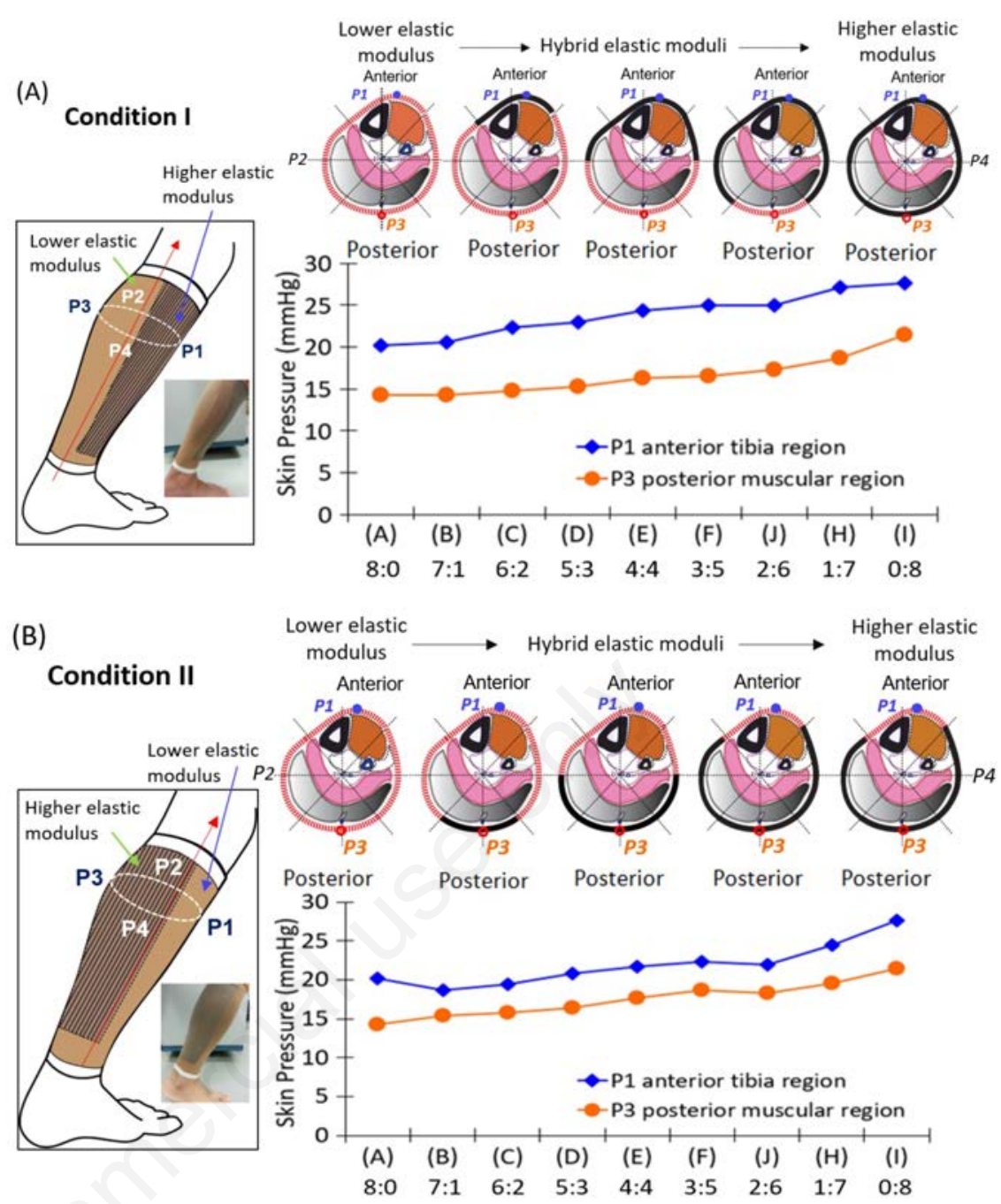

Figure 1. Skin pressure exerted by HCSs with nine types of hybrid elastic moduli. A) Condition I: segments with higher elastic moduli (shorter stretch) at anterior side, and segments with lower elastic moduli (longer stretch) at posterior region; B) Condition II: segments with lower elastic moduli (longer stretch) at anterior side, and segments with higher elastic moduli (short-stretch) at posterior calf.
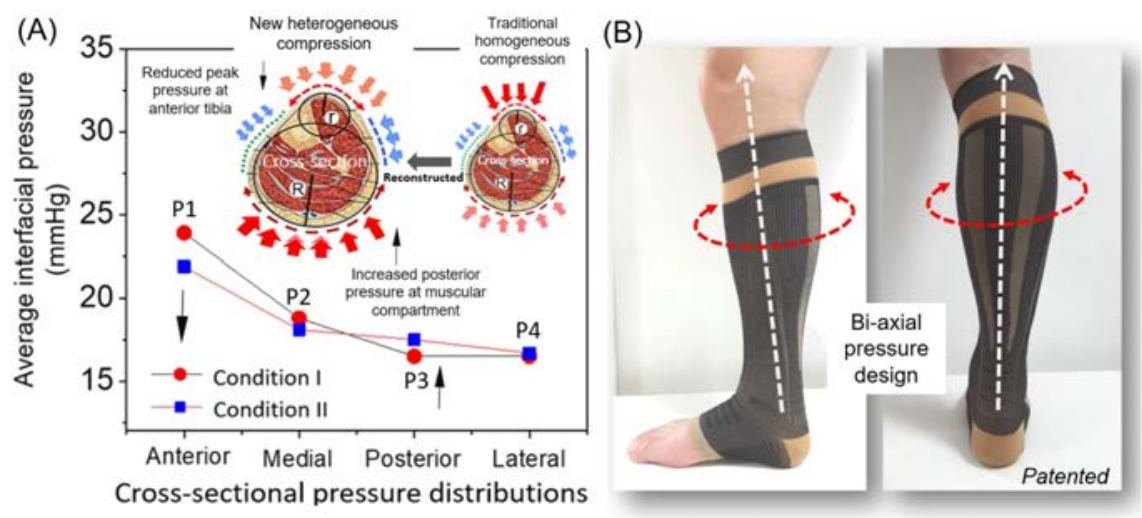

Figure 2. A) Cross-sectional pressure variations of calf by HCSs under conditions I and II. A new cross-sectional profile with reduced anterior pressure and increased posterior pressure was demonstrated; B) a new compression stocking with hybrid low-high elastic moduli (2:6) set in condition II was developed to deliver a controlled bi-axial pressure mode along lower limb. 
pressure at a given sized limb. The hybrid elastic moduli segments set at condition II demonstrated to reduce the anterior tibia pressure but increase posterior muscular pressure. The design of specimen $\mathrm{G}$ with low-high elastic moduli panel ratio of 2:6 (i.e. $25 \%: 75 \%$ ) set in condition II could be a possible solution to fulfill the findings reported by Stolk et al. ${ }^{16}$ They found that only $35 \%$ on average of leg perimeter near the calf expanded at posterior side, whereas $65 \%$ including anterior region did not expand at all in kinematic movement. In the designed HCSs, the segments with higher elastic moduli set at posterior region against muscular expansion shows the promising capability to augment pumping action during muscular contraction and relaxation in dynamic use.

Calf muscular pump plays significant role in promotion of venous return; however, the anatomy of the calf puts it at increased risk for side effects induced by uneven pressure delivery. Build-up of pressure in anterior bone area cannot decompress to around compartment due to higher tissue stiffness $\left(36.4-44.9 \mathrm{kgf} / \mathrm{mm}^{2}\right)$. The anterior tibia crest with less radius of curvature may cause high peak pressure and be more subjected to skin damage and pressure sore in sustained compression therapy. The skin pressure at posterior muscular region could be deficient due to absorption and dissipation of pressure by surrounding tissues with less stiffness (16.5-25.4 $\mathrm{kgf} / \mathrm{mm}^{2}$ ). The new heterogeneous compression shells provide an optional method to proactively control and reshape crosssectional pressure at calf.

\section{Conclusions}

The new HCSs demonstrated an important mechanism, that is, heterogeneous compression shells can reconstruct pressure profiles on irregular body to improve pressure function, which is considered to be a promising mode to reduce peak focal pressure at anterior tibia crest but augment muscular pumping action in dynamic wear. Based on this results, a new generation of compression stockings with bi-axial pressure function has been developed to deliver both controlled more even cross-sectional pressure horizontally and gradient skin pressure along the lower limb lengthwise. Further studies need to be carried out to optimize hybrid elastic moduli for multiple pressure levels for treatment of CVI symptoms.

\section{References}

1. Shammeri OA, Aihamdan N, Aihothaly B, et al. Chronic venous insufficiency: prevalence and effect of compression stockings. Int $\mathrm{J}$ Health Sci 2014;8:231-6.

2. Hohlbaum GG. Zur geschichte der kompressions therapie. I. Phlebol Proktol 1987;16:241-55.

3. Robertson BF, Thomson $\mathrm{CH}$, Siddiqui H. Side effects of compression stockings: a case report. $\mathrm{Br} \mathrm{J}$ Gen Pract 2014;64:316-7.

4. Hooke's Law. In: Pautrup B ed.), Physics of continuous matter: exotic and everyday phenomena in the macroscopic world. 2nd ed. CRF Press; 2011. pp.125-138.

5. Partsch H. Compression therapy: clinical and experimental evidence. Ann Vasc Dis 2012;5:416-22.

6. Mosti G, Partsch H. Duplex scanning to evaluate the effect of compression on venous reflux. Int Angiol 2010;29:41620.

7. Lattimer CR, Kalodiki E, Kafeza M, et al. Quantifying the degree graduated elastic compression stockings enhance venous emptying. Eur J Vasc Endovasc Surg 2014;47:75-80.

8. Mosti G, Partsch H. Improvement of venous pumping function by double progressive compression stockings: higher pressure over the calf is more important than a graduated pressure profiles. Eur J Vasc Endovasc Surg 2014;47:545-9.

9. Tandler SF. Challenges faced by healthcare professionals in the provision of compression hosiery to enhance compliance in the prevention of venous leg ulceration. EWMA J 2016;16:29-33.

10. Kahn SR, Shapiro S, Wells PS, et al. Compression stockings to prevent postthrombotic syndrome: a randomised placebo-controlled trial. Lancet 2013.

11. Raju S, Hollis K, Neglen P. Use of compression stockings in chronic venous disease: patient compliance and efficacy. Ann Vasc Surg 2007;21:790-5.

12. Strölin A, Häfner HM, Jünger $M$. Biophysical characteristics of medical compression stockings. Phlebologie 2007;36:197-204.

13. Perrin M. Skin necrosis as a complication of compression in the treatment of venous disease and in prevention of venous thromboembolism. Phlebolymphology 2008;15:27-30.

14. Robertson B, Thomson C, Siddiqui H. Side effects of compression stockings: a case report. Br J Gen Pract 2014;64: 316-7.

15. Thomas S. The use of the Laplace equation in the calculation of sub-bandage pressure. EWMA J 2003;3:21-3.

16. Stolk R, Wegen van der-franken CPM, Neumann HAM. A method for measuring the dynamic behaviour of medical compression hosiery during walking. Dermatol Surg 2004;30:729-36. 